\title{
Revisiting theoretical and causal explanations for the bilingual advantage in executive functioning
}

Vera Kempe, Neil W. Kirk and Patricia J. Books

This is the accepted manuscript (C) 2015, Elsevier

Licensed under the Creative Commons Attribution-NonCommercialNoDerivatives 4.0 International

http://creativecommons.org/licenses/by-nc-nd/4.0/

(cc) EY-NC-ND

The published article is available from doi: 10.1016/j.cortex.2015.07.021 
Revisiting theoretical and causal explanations

for the bilingual advantage in executive functioning

\author{
Vera Kempe \\ Neil W. Kirk \\ Abertay University, Dundee \\ Patricia J. Brooks \\ College of Staten Island and The Graduate Center, CUNY \\ Acknowledgement: Parts of this research were supported by Leverhulme Trust \\ Research Project Grant \# RPG-375 to Vera Kempe and by various grants from the \\ journal Language Learning to Patricia J. Brooks and Vera Kempe.
}


The hypothesis of a bilingual advantage in executive functioning derives from models of bilingual lexical access, postulating that to access lexical representations in one language, the target language task schema must be selectively activated and lexical competitors in the non-target language reactively inhibited (Green, 1998). Bilinguals' routine deployment of selective attention and inhibition in the domain of language is assumed to hone these executive processes to such an extent that far transfer (Barnett \& Ceci, 2002) of attentional control to non-linguistic domains ultimately occurs. Hence, bilinguals are expected to outperform monolinguals on non-linguistic, executive functioning tasks. Paap, Johnson and Sawi (2015) provide several compelling arguments for why the empirical evidence in support of a bilingual advantage in executive functioning is shaky. We agree that their arguments constitute valid grounds for skepticism, but rather than dismissing the hypothesis entirely, we offer further arguments for why findings have been inconsistent and why, even when a bilingual advantage is evident, the commonly provided causal interpretation might be wrong.

The first argument is that many monolinguals might not differ fundamentally from bilinguals in terms of their reliance on executive control processes during lexical access. Many monolinguals routinely switch between different varieties of their native language, such as accents, dialects, or sociolects, depending on interlocutor and context. Models of lexical representation and lexical access have largely ignored the existence of different linguistic varieties (but see La Heij, 2005). To examine whether bidialectal speakers exhibit similar conflict-resolution processes during lexical access as bilinguals, we designed a dialect-switching task requiring participants to name pictures using either a standard or a dialect variety (Kirk, Declerck, Scott-Brown, Kempe \& Philipp, 2014). We compared naming latencies of monodialectal speakers 
of English with limited exposure to Dundonian, a regional Scots dialect, with bidialectals who routinely use Dundonian in addition to the standard variety of English spoken in Scotland. The results fully replicated findings from bilinguals (Meuter \& Allport, 1999): Naming latencies were longer when participants switched between dialect and standard, and cognates were named faster than non-cognates (Christoffels, Firk \& Schiller, 2007), suggesting that both varieties are active during bidialectal lexical access. Furthermore, bidialectal speakers who routinely used both varieties exhibited symmetrical switch costs, just like balanced bilinguals (Costa \& Santesteban, 2004), whereas monodialectal speakers with limited Dundonian exposure exhibited asymmetrical switch costs such that higher costs were associated with switching into the dominant standard variety. This latter result mirrors findings for unbalanced bilinguals where asymmetrical switch costs reflect task-set inertia because more time is required to overcome the stronger inhibition required to block out the dominant language schema on previous trials (Green, 1998).

These results, also replicated with German bidialectals (Kirk et al., 2014), point to the possibility that architectures of lexical representation and mechanisms of lexical access might be fundamentally similar in bidialectals and bilinguals. Consequently, no differences would be expected in executive functioning tasks if bilinguals were compared to bidialectal speakers who, without a sensitive measure of dialect use, would self-identify as monolingual. Given that different research groups not only have access to different populations of bilinguals, but also to different populations of monolinguals, the use of different linguistic varieties in monolinguals may influence patterns of results. This might account for the fact that research group emerged as a significant moderator of effect sizes in a meta-analysis of conflictresolution tasks evaluating the bilingual advantage hypothesis (Donnelly, Brooks \& 
Homer, 2015). If corroborated by further research, the suggestion that mechanisms of bidialectal and bilingual lexical access are fundamentally the same calls into question the basic assumption of reduced executive involvement in monolingual lexical access, and may account for some of the failures to replicate the bilingual advantage, as documented by Paap et al. (2015).

The second argument is that links between bilingualism and executive functioning may, at least in part, arise because superior executive functioning is a cause rather than a consequence of bilingualism. Research on individual differences in adult second language learning consistently shows that non-verbal intelligence and working memory capacity are strong predictors of learners' success (e.g., Brooks \& Kempe, 2013; Miyake \& Friedman, 1998). Measures of psychometric intelligence and working memory capacity, albeit different from the measures of conflict resolution used to demonstrate a bilingual advantage, are nonetheless linked to various executive functions (Duncan, Emslie, Williams, Johnson \& Freer, 1996; Friedman, Miyake, Corley, Young, DeFrie \& Hewitt, 2006). If these variables contribute to language learning success then, in the context of immigration, individual differences in executive functioning might explain why some immigrants show limited success in attaining bilingual proficiency, e.g., by failing to retain proficiency in their heritage language while shifting dominance to the adopted language.

Moreover, given that individuals tend to seek out environments and pursuits that best suit their cognitive strengths (Haworth, Wright, Luciano et al., 2010), superior executive functioning might predict which individuals are likely to seek out language learning opportunities in the first place. To obtain some preliminary evidence for this claim, we pooled data of 396 research participants from language learning experiments conducted in our laboratory over a period of about 10 years. 
These participants completed Cattell's Culture Fair test of non-verbal intelligence (Cattell \& Cattell, 1973) and reported how many languages they had learned in the past, predominantly in educational settings (Brooks \& Kempe, 2013; Brooks, Kempe \& Sionov, 2006; Kempe \& Brooks, 2008; Kempe, Brooks \& Kharkhurin, 2010). After controlling for age, non-verbal intelligence accounted for a significant $2.2 \%$ of variance in how many languages participants had previously learned $(\beta=1.6, \mathrm{p}<$ .001). It seems just as plausible to interpret this correlation as evidence that individuals with higher non-verbal intelligence more often seek out opportunities to engage in language learning (both in naturalistic and in formal settings) than to assume that taking up languages in school or college boosts their non-verbal intelligence test scores. Thus, in addition to the compelling arguments against the bilingual advantage hypothesis provided by Paap et al. (2015) we point out that alternative causal interpretations for a link between executive functioning and bilingualism are conceivable. 
References:

Barnett, S. M., \& Ceci, S. J. (2002). When and where do we apply what we learn? A taxonomy for far transfer. Psychological Bulletin, 128, 612-637.

Brooks, P. J., Kempe, V. (2013) Individual differences in adult foreign language learning: The mediating effect of meta-linguistic awareness. Memory \& Cognition, 41, 281-296.

Brooks, P. J., Kempe, V. \& Sionov, A. (2006). The role of learner and input variables in learning inflectional morphology. Applied Psycholinguistics, 27, 185209.

Cattell, R. B. \& Cattell, H. E. P. (1973). Measuring Intelligence with the Culture-Fair Tests. Champaign, IL: Institute for Personality and Ability Testing.

Costa, A., \& Santesteban, M. (2004). Lexical access in bilingual speech production: Evidence from language switching in highly proficient bilinguals and L2 learners. Journal of Memory and Language, 50(4), 491-511.

Donnelly, S., Brooks, P. J., \& Homer, B. (2015). Examining the bilingual advantage on conflict resolution tasks: A meta-analysis. In Proceedings of the 37th Annual Conference of the Cognitive Science Society.

Duncan, J., Emslie, H., Williams, P., Johnson, R., \& Freer, C. (1996). Intelligence and the frontal lobe: The organization of goal-directed behavior. Cognitive Psychology, 30(3), 257-303.

Friedman, N. P., Miyake, A., Corley, R. P., Young, S. E., DeFries, J. C., \& Hewitt, J. K. (2006). Not all executive functions are related to intelligence. Psychological Science, 17(2), 172-179.

Haworth, C. M. A., Wright, M. J., Luciano, M., Martin, N. G., De Geus, E. J. C., Van Beijsterveldt, C. E. M., ... \& Plomin, R. (2010). The heritability of general 
cognitive ability increases linearly from childhood to young adulthood. Molecular Psychiatry, 15(11), 1112-1120.

Kempe, V. \& Brooks, P. J. (2008). Second language learning of complex inflectional systems. Language Learning, 54(4), 703-746.

Kempe, V., Brooks, P. J. \& Kharkhurin, A. (2010). Cognitive predictors of generalization of Russian grammatical gender categories. Language Learning, 60, $127-153$.

Kirk, N. W., Declerck, M., Scott-Brown, K., Kempe, V. \& Philipp, A. (2014). Cognitive cost of switching between standard and dialect varieties. Poster presented at the 20th Conference on Architectures and Mechanisms for Language Processing. Edinburgh, UK.

La Heij, W. 2005: Selection processes in monolingual and bilingual lexical access. In J. Kroll, J. and A. de Groot (Eds.), Handbook of bilingualism (pp. 289307). Oxford: Oxford University Press.

Meuter, R. F., \& Allport, A. (1999). Bilingual language switching in naming: Asymmetrical costs of language selection. Journal of Memory and Language, 40(1), $25-40$.

Miyake, A., \& Friedman, N. P. (1998). Individual differences in second language proficiency: Working memory as language aptitude. In A. F. Healy \& L. E. Bourne (Eds.), Foreign language learning: Psycholinguistic studies on training and retention (pp. 339-364). Mahwah, NJ: Erlbaum

Paap, K. R., Johnson, H. A. \& Sawi, O. (2015). Bilingual advantages in executive functioning either do not exist or are restricted to very specific and undetermined circumstances. Cortex. 